Supporting Information

\title{
Age-dependent glycomic response to the 2009 pandemic H1N1 influenza virus and its association with disease severity
}

Shuhui Chen', Brian Kasper ${ }^{1}$, Bin Zhang, Lauren P. Lashua ${ }^{3}$, Ted M. Ross ${ }^{4}$, Elodie Ghedin $^{3,5}$ and Lara K. Mahal ${ }^{1,6 ¥}$

${ }^{1}$ Biomedical Research Institute, Department of Chemistry, New York University, NY, 10003, USA;

${ }^{2}$ Department of Genetics and Genomic Sciences, Mount Sinai Center for Transformative Disease Modeling, Icahn Institute of Genomics and Multiscale Biology, Icahn School of Medicine at Mount Sinai, NY, 10029, USA;

${ }^{3}$ Center for Genomics \& Systems Biology, Department of Biology, New York University, NY, 10003, USA;

${ }^{4}$ Center for Vaccines and Immunology, University of Georgia, GA, 30602, USA;

${ }^{5}$ Systems Genomics Section, Laboratory of Parasitic Diseases, NIAID/NIH, Bethesda, MD, 20894, USA; 
${ }^{6}$ Department of Chemistry, University of Alberta, Edmonton, AB, T6G 2G2, CANADA.

${ }^{¥}$ Corresponding author: Dr. Lara K. Mahal, Canada Excellence Research Chair in Glycomics orcid.org/0000-0003-4791-8524; Phone: 780-492-5847; Email: $\underline{\text { kmahal@ualberta.ca }}$ 


\section{Table of Contents}

Figure S1. Boxplots of $\alpha-2,6$-sialoside signal by age in uninfected ferrets.

Figure S2. Boxplots of $\alpha$-2,3-sialoside levels by age in uninfected ferrets.

Figure S3. Boxplots of mucins expressions by age in uninfected ferrets.

Figure S4. Boxplots of Lewis structures by age in uninfected ferrets.

Figure S5. Boxplots of high/oligo-mannose levels by age in uninfected ferrets.

Figure S6. Boxplots of Pneumonia Composite Score (PCS) as a function of weight loss quartiles in adult and aged ferrets.

Figure S7. Aged ferrets had delayed response to influenza infection.

Figure S8. Impact of influenza infection on $\alpha-2,6$-sialosides in adult and aged ferrets.

Figure S9. Impact of influenza infection on $\alpha$-2,3-sialosides in adult and aged ferrets.

Figure S10. Impact of influenza infection on $O$-linked glycans in aged ferrets.

Figure S11. Dynamic changes in $O$-linked glycans are observed upon infection in aged ferrets.

Figure S12. Comparison of severity metrics used in adult and aged ferrets.

Figure S13. Glycan changes are observed early in the time-course study of newly weaned ferrets.

Table 1. Lectins used in microarrays

Table 2. Lectin microarray information-MIRAGE format. 

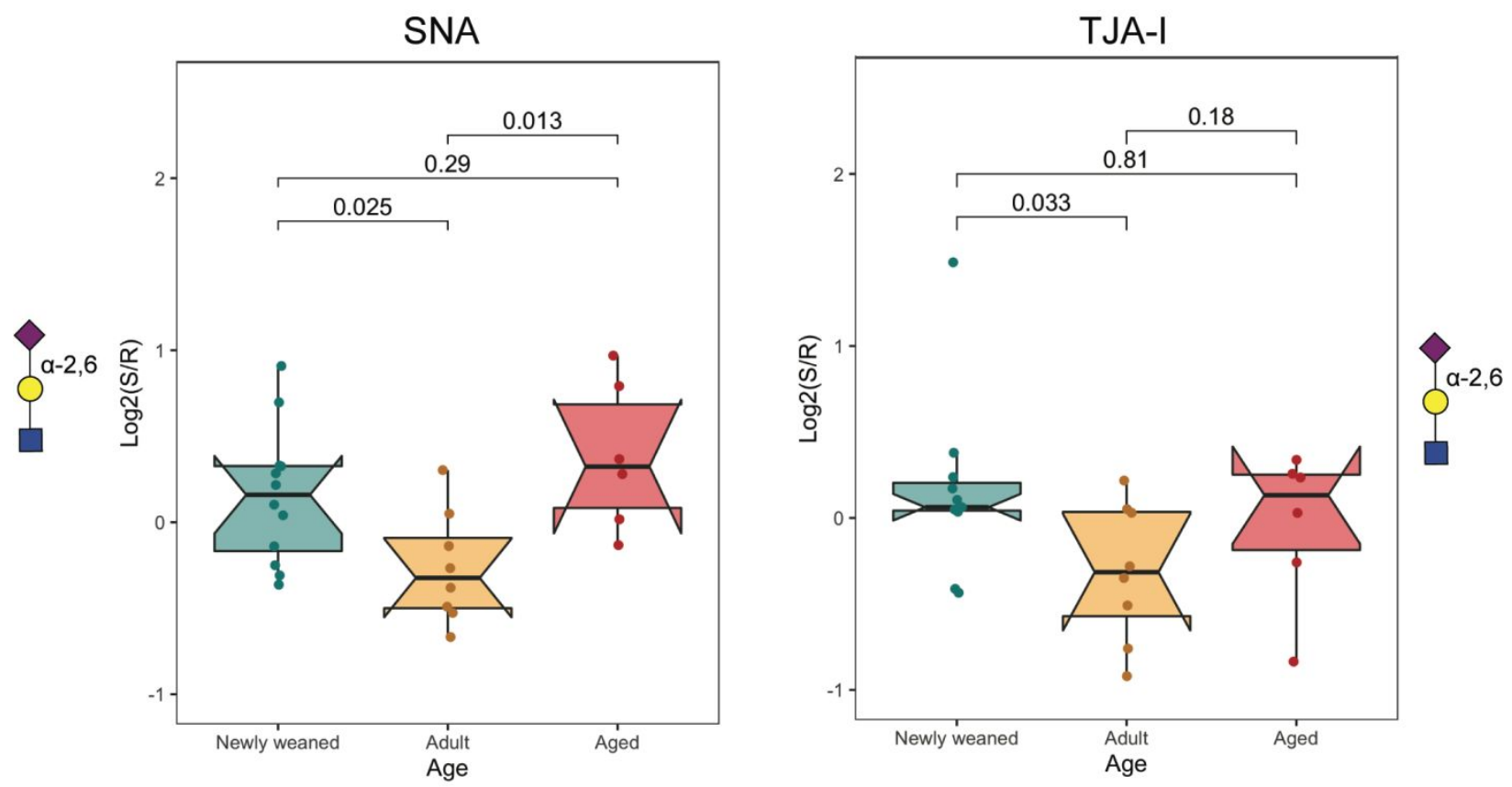

Figure S1. Boxplots of $\boldsymbol{\alpha}-\mathbf{2 , 6}$-sialoside signal by age in uninfected ferrets. Boxplots of binding by $\alpha 2,6$-sialic acid lectins (SNA and TJA-I). Median normalized $\log _{2}$ ratios (Sample $(\mathrm{S}) /$ Reference $(\mathrm{R})$ ) are plotted by age (Newly weaned: cyan; Adult: orange; Aged: red). P-value from Wilcoxon's t-test is shown for indicated pairs. Glycans bound by lectins are shown in the Symbolic Nomenclature for Glycomics (SNFG) at the side of the boxplots. 

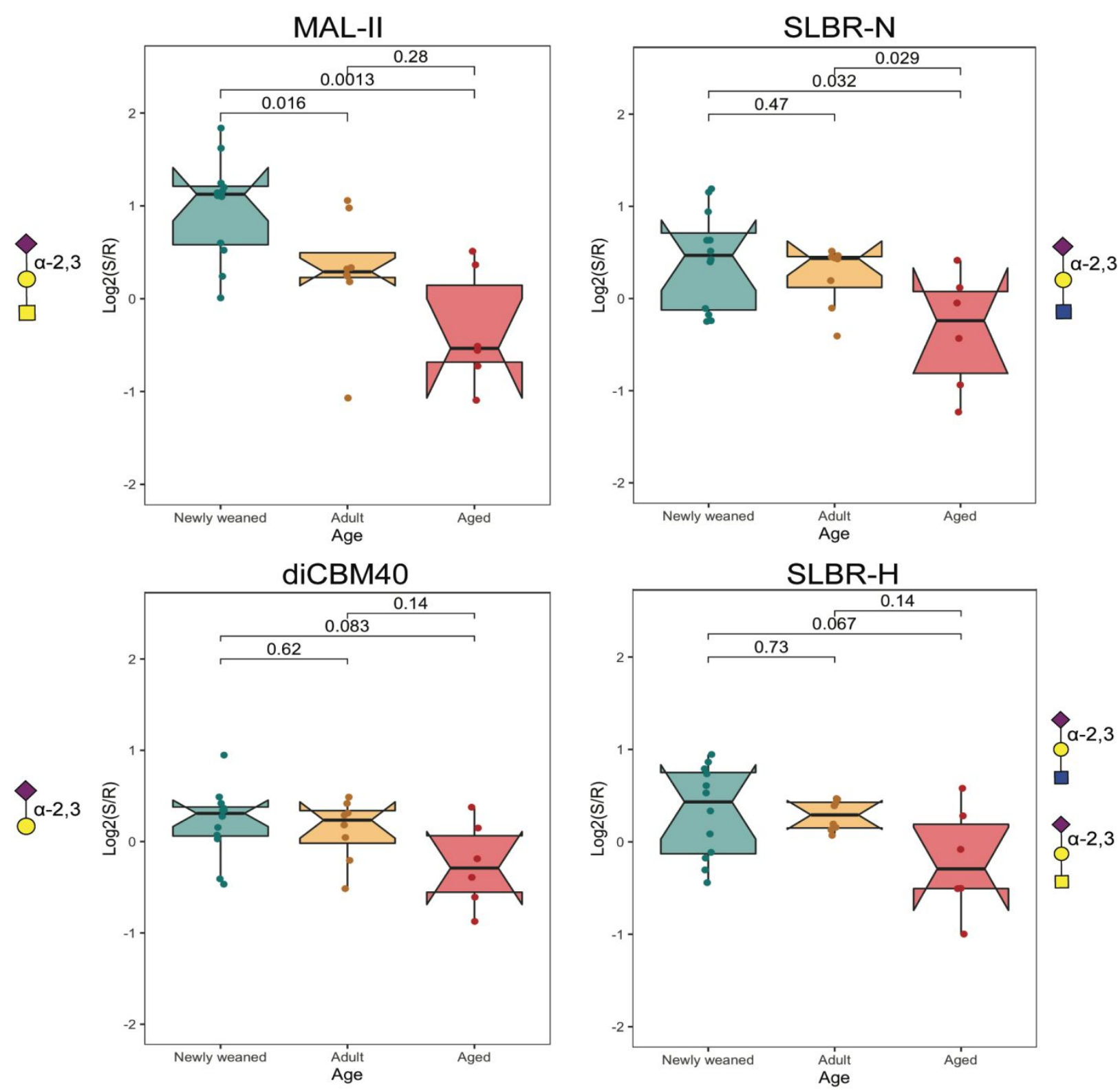

Figure S2. Boxplots of $\boldsymbol{\alpha}$-2,3-sialoside levels by age in uninfected ferrets. Boxplots of binding by $\alpha 2,3$-sialic acid lectins (MAL-II, SLBR-N, diCBM40, SLBR-H). Median normalized $\log _{2}$ ratios (Sample $(\mathrm{S}) /$ Reference $(\mathrm{R})$ ) are plotted by age (Newly weaned: cyan; Adult: orange; Aged: red). P-value from Wilcoxon's t-test is shown for indicated pairs. Glycans bound by lectins are shown in SNFG at the side of the boxplots. 

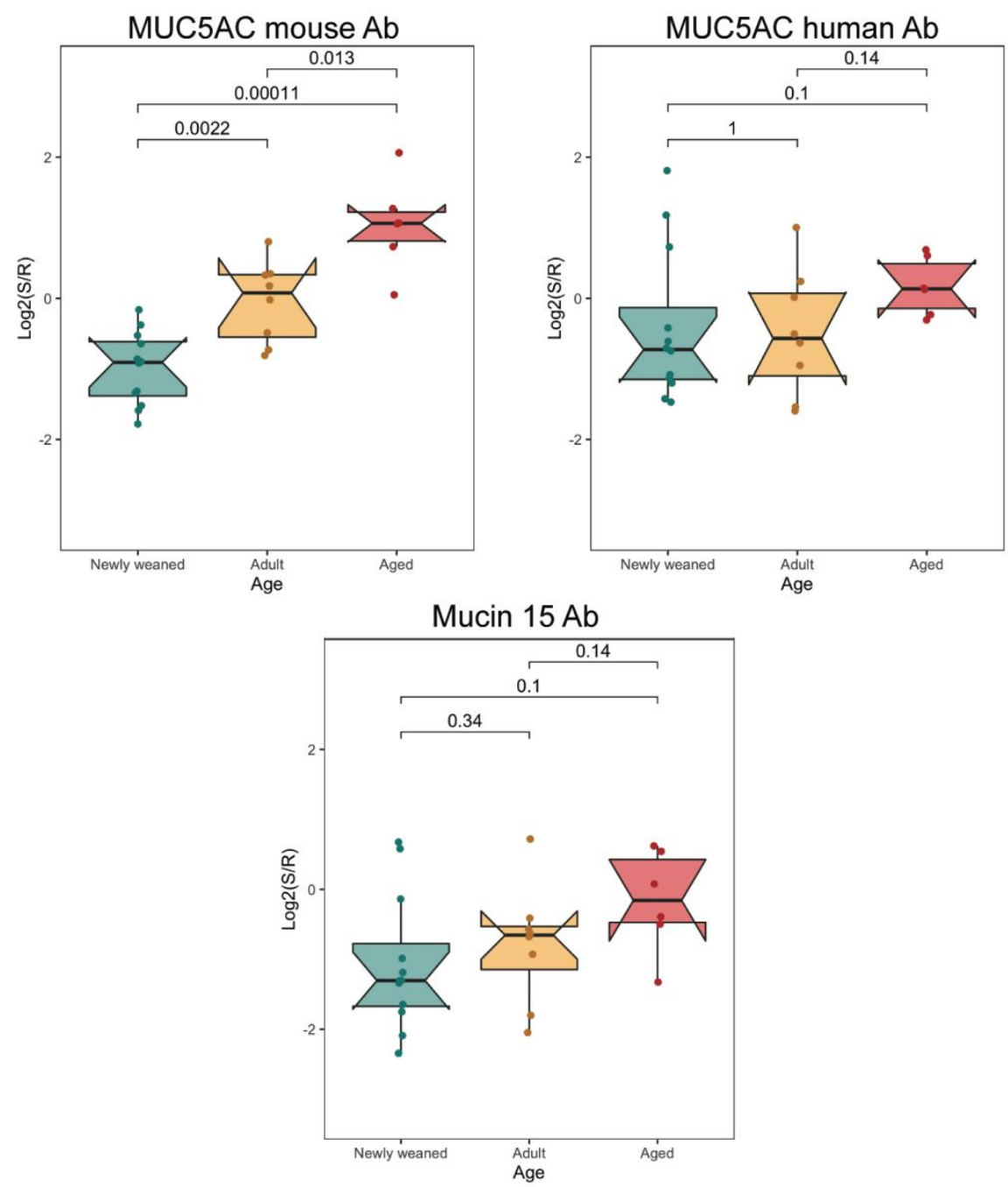

Figure S3. Boxplots of mucins expressions by age in uninfected ferrets. Boxplots of binding by mucin antibodies (MUC5AC mouse Ab, MUC5AC human Ab and Mucin 15 Ab). Median normalized $\log _{2}$ ratios (Sample (S)/Reference(R)) are plotted by age (Newly weaned: cyan; Adult: orange; Aged: red). P-value from Wilcoxon's t-test is shown for indicated pairs. 

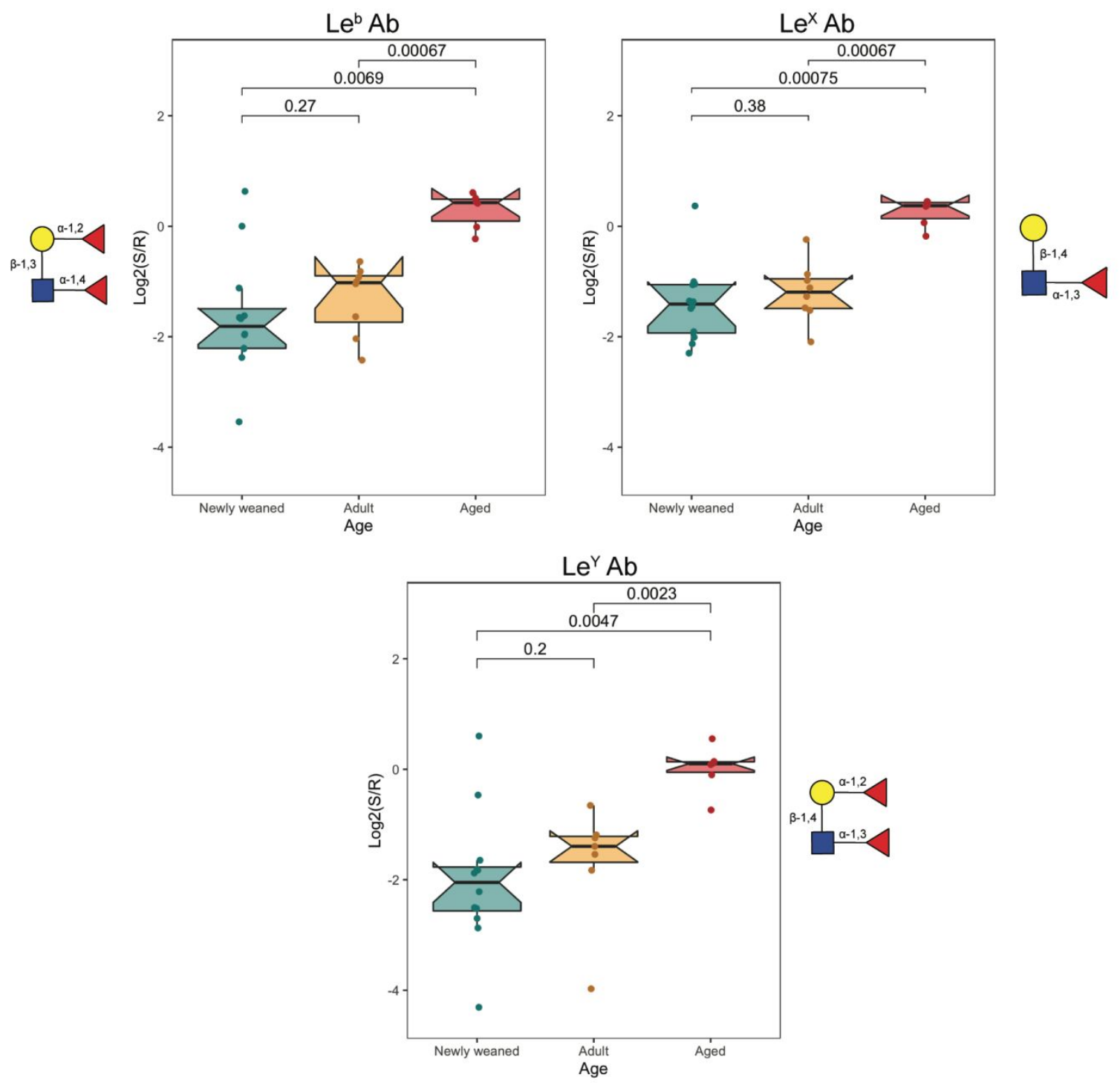

Figure S4. Boxplots of Lewis structures by age in uninfected ferrets. Boxplots of binding by Lewis structures antibodies ( $\mathrm{Le}^{\mathrm{b}} \mathrm{Ab}, \mathrm{Le}^{\mathrm{X}} \mathrm{Ab}$ and $\mathrm{Le}^{\mathrm{Y}} \mathrm{Ab}$ ). Median normalized $\log _{2}$ ratios (Sample (S)/Reference(R)) are plotted by age (Newly weaned: cyan; Adult: orange; Aged: red). P-value from Wilcoxon's t-test is shown for indicated pairs. Glycans bound by lectins are shown in SNFG at the side of the boxplots. 

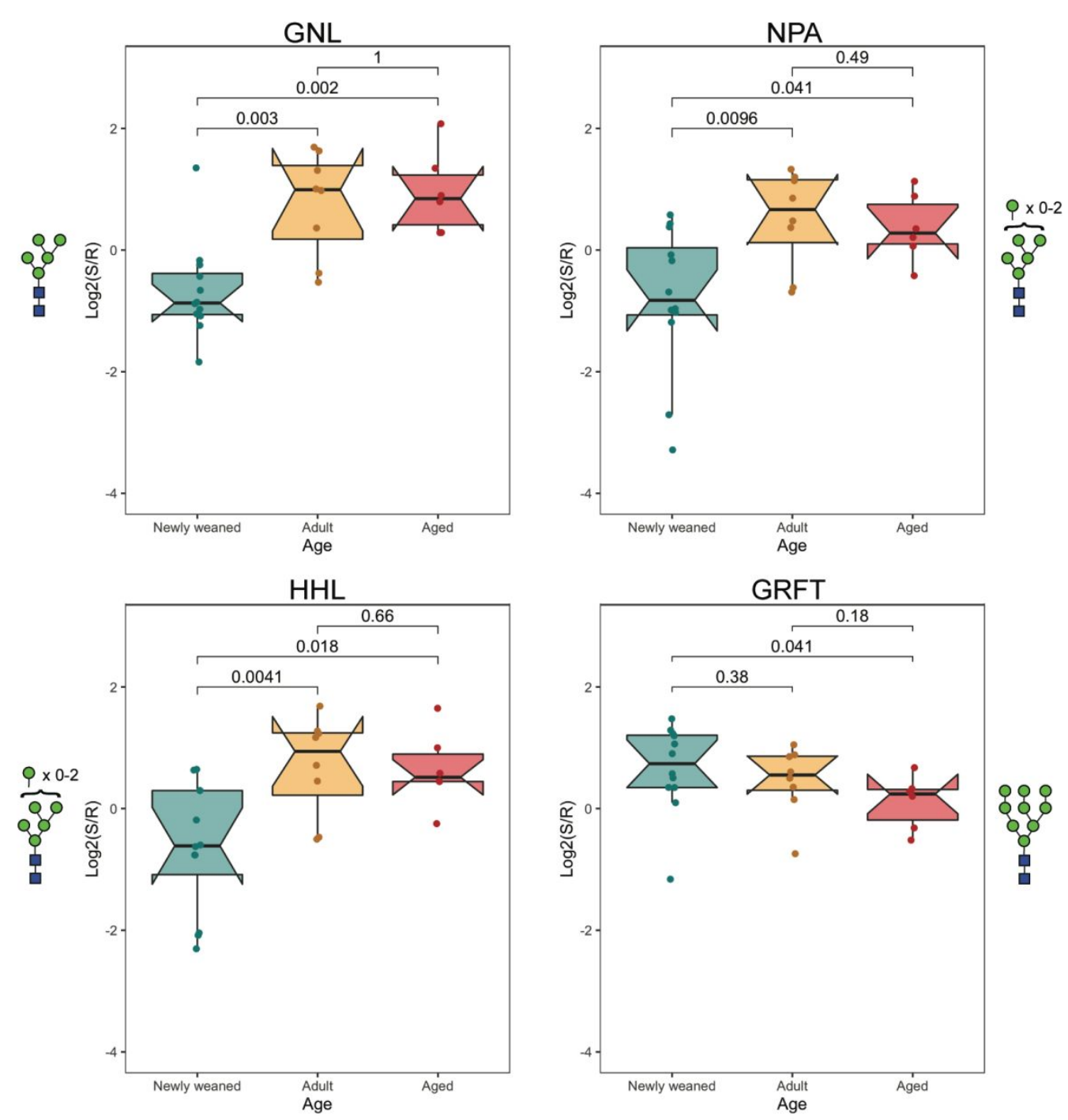

Figure S5. Boxplots of high/oligo-mannose levels by age in uninfected ferrets. Boxplots of binding by high/oligo-mannose lectins (GNL, NPA, HHL and GRFT). Median normalized $\log _{2}$ ratios (Sample $(\mathrm{S}) /$ Reference $(\mathrm{R})$ ) are plotted by age (Newly weaned: cyan; Adult: orange; Aged: red). P-value from Wilcoxon's t-test is shown for indicated pairs. Glycans bound by lectins are shown in SNFG at the sides of the boxplots. 

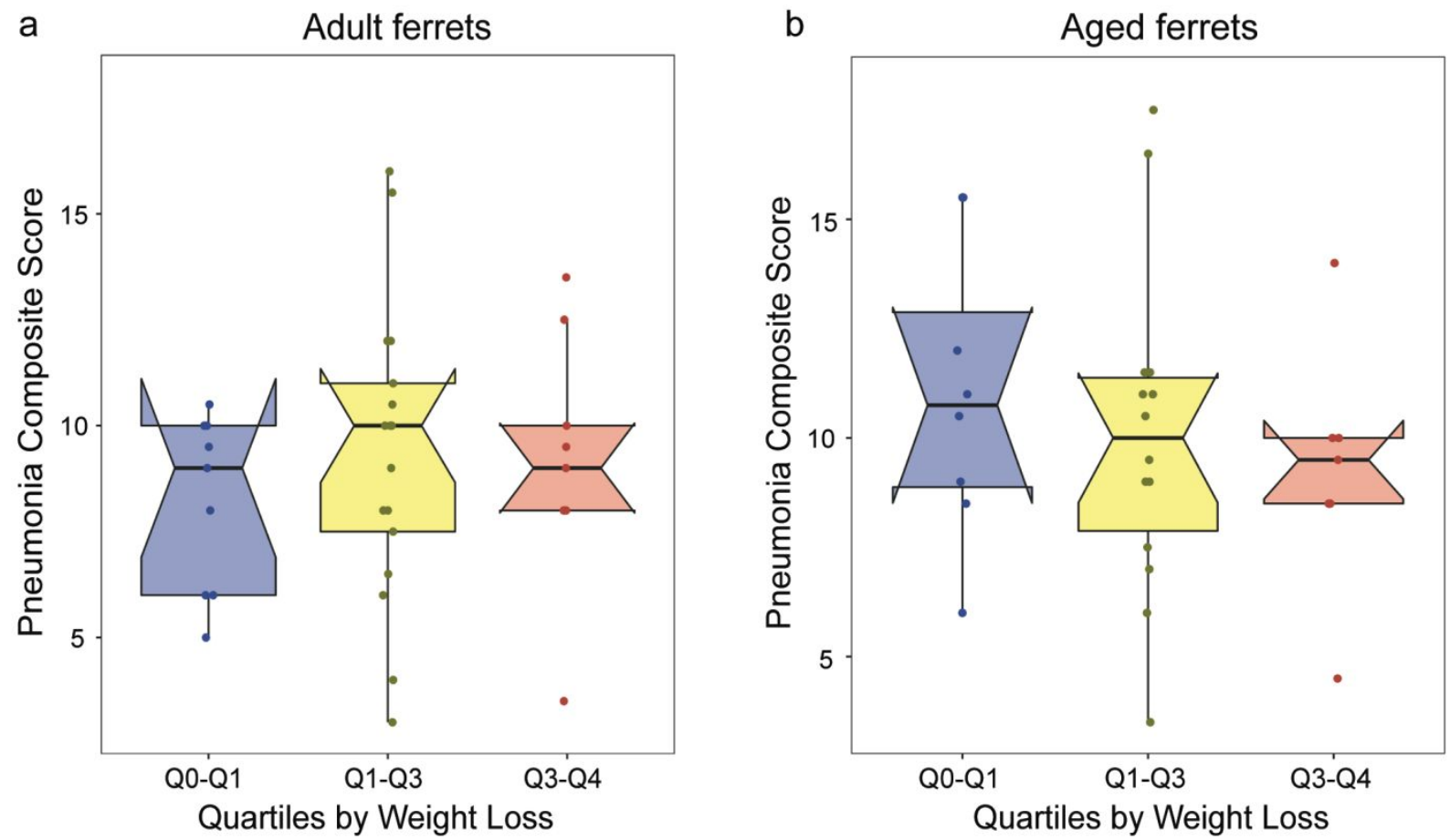

Figure S6. Boxplots of Pneumonia Composite Score (PCS) as a function of weight loss quartiles in adult and aged ferrets. Q0-Q1: $1^{\text {st }}$ quartile (blue), Q1-Q3: middle quartiles (yellow), Q3-Q4: last quartile (red) of weight loss percent. a) Boxplot analysis of PCS correlation to weight loss in adult ferrets at day 8 post-infection with H1N1pdm09. (Q0-Q1, $\mathrm{n}=$ 9 ferrets; Q1-Q3, $\mathrm{n}=17$; Q3-Q4, $\mathrm{n}=9$ ). b) Boxplot analysis of correlation in aged ferrets at day 8 post-infection (Q0-Q1, $\mathrm{n}=8$ ferrets; Q1-Q3, $\mathrm{n}=14$; Q3-Q4, $\mathrm{n}=7$ ). 


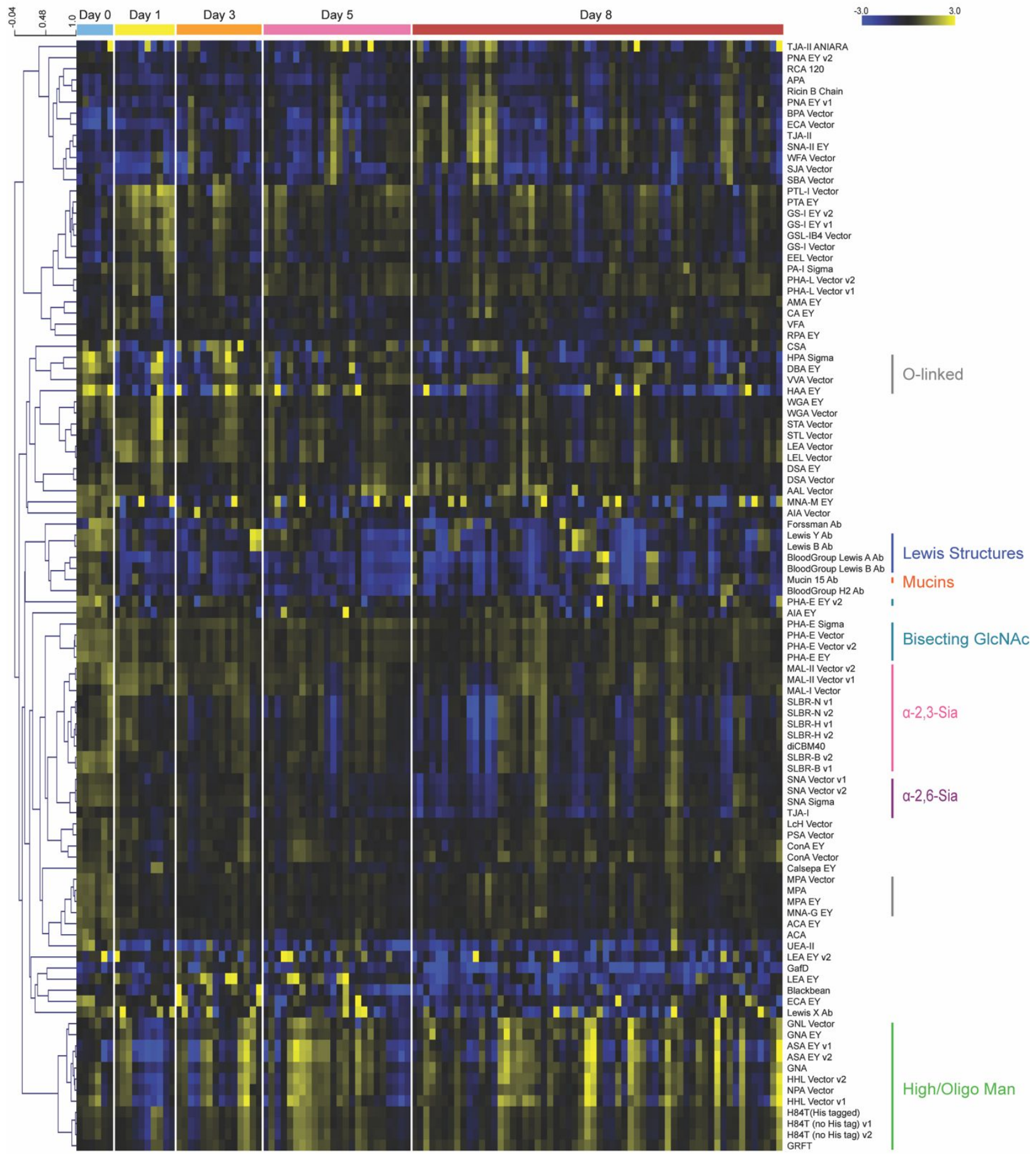

Figure S7. Aged ferrets had delayed response to influenza infection. Median normalized $\log _{2}$ ratios (Sample $(\mathrm{S}) /$ Reference $(\mathrm{R})$ ) of aged ( $>5.5$ years) ferret lung samples were ordered by days post infection. Uninfected (blue), $\mathrm{n}=3$; day 1 (yellow), $\mathrm{n}=5$; day 3 (orange), $\mathrm{n}=7$; day 5 (pink), $\mathrm{n}=12$; day $8(\mathrm{red}), \mathrm{n}=29.2$ samples per ferret. Yellow, $\log _{2}(\mathrm{~S})>\log _{2}(\mathrm{R})$; blue, $\log _{2}(\mathrm{R})>\log _{2}(\mathrm{~S})$. Lectins binding $\alpha-2,3$-sialosides (pink), $\alpha-2,6$-sialosides (purple), high/oligo-mannose (green), bisecting GlcNAc (turquoise), $O$-linked glycans (charcoal), mucins (orange) and Lewis structures (blue) are highlighted on the right of the heatmap. 

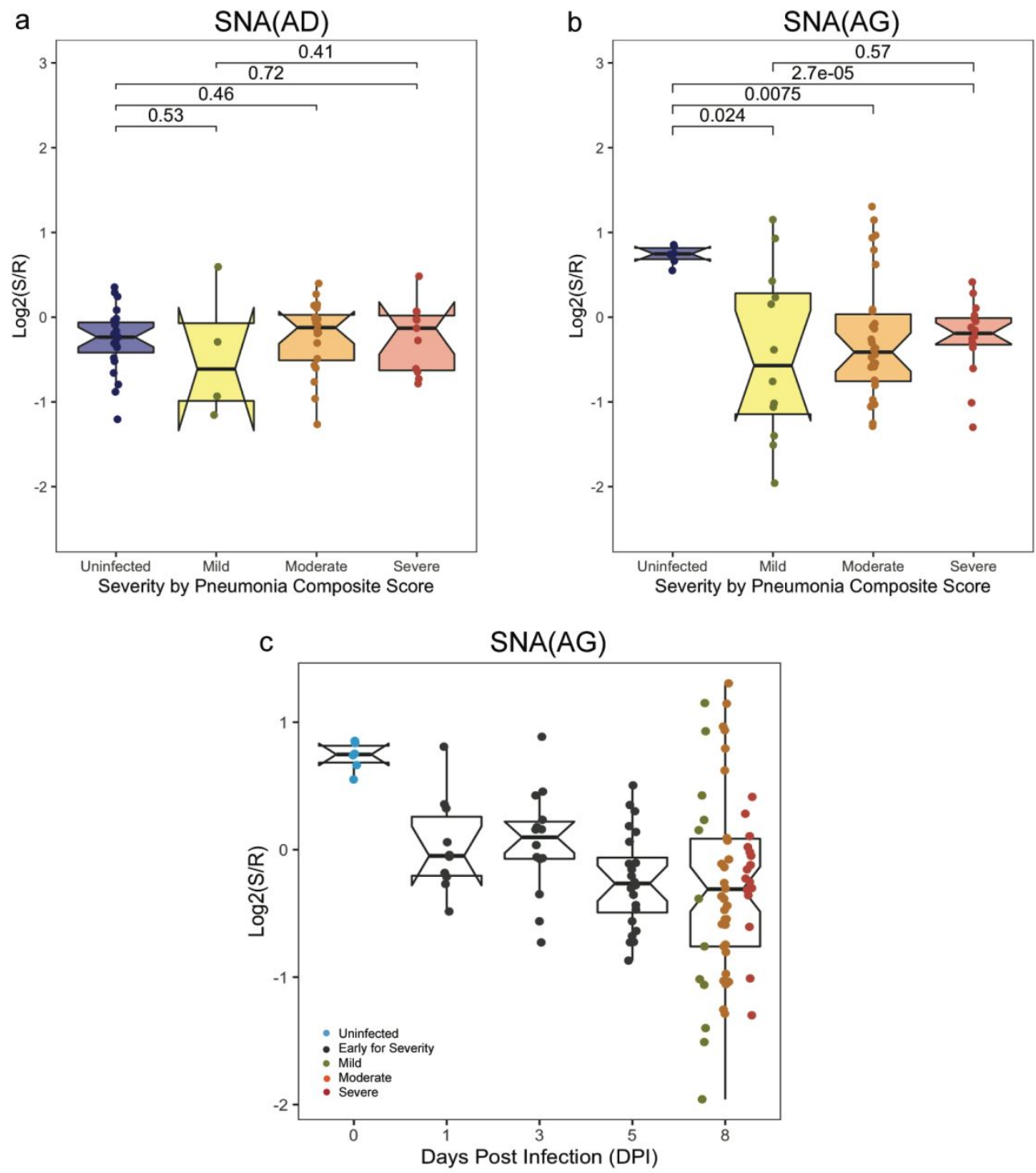

Figure S8. Impact of influenza infection on $\alpha-2,6$-sialosides in adult and aged ferrets. a) Severity study of $\alpha-2,6$-sialic acids probed by SNA at day 8 post-infection in adult ferrets (AD) is shown. Boxplots of median normalized $\log _{2}$ ratios (Sample (S)/Reference(R)) of ferret lung samples were ordered by severity defined by pneumonia composite score (PCS). Uninfected (blue): $\mathrm{n}=4,4$ samples per ferret. Day 8 post-infection: Mild (yellow), $\mathrm{n}=2,2$ samples per ferret; Moderate (orange), $\mathrm{n}=11,2$ samples per ferret; Severe (red), $\mathrm{n}=6,2$ samples per ferret. b) Severity study of $\alpha-2,6$-sialic acids probed by SNA at day 8 post-infection in aged ferrets (AG) is shown. Boxplots of median normalized $\log _{2}$ ratios (Sample $(\mathrm{S}) /$ Reference(R)) of ferret lung samples were ordered by severity defined by PCS. Uninfected (blue): $n=3,2$ samples per ferret. Day 8 post-infection (2 samples per ferret): Mild (yellow), $\mathrm{n}=6$; Moderate (orange), $\mathrm{n}=15$; Severe (red), $\mathrm{n}=8$. P-value from Wilcoxon's t-test is shown for indicated pairs. c) Time-course analysis of $\alpha-2,6$-sialic acid levels probed by SNA following H1N1pdm09 infections $(\mathrm{t}=0,1,3$, 5 and 8 days) in aged animals (AG). Boxplot of median normalized $\log _{2}$ ratios (Sample $(\mathrm{S}) /$ Reference $(\mathrm{R}))$ is shown. Disease severity is indicated by color (Uninfected: blue; Early for Severity: black; Mild: dark yellow; Moderate: orange, Severe: red). 

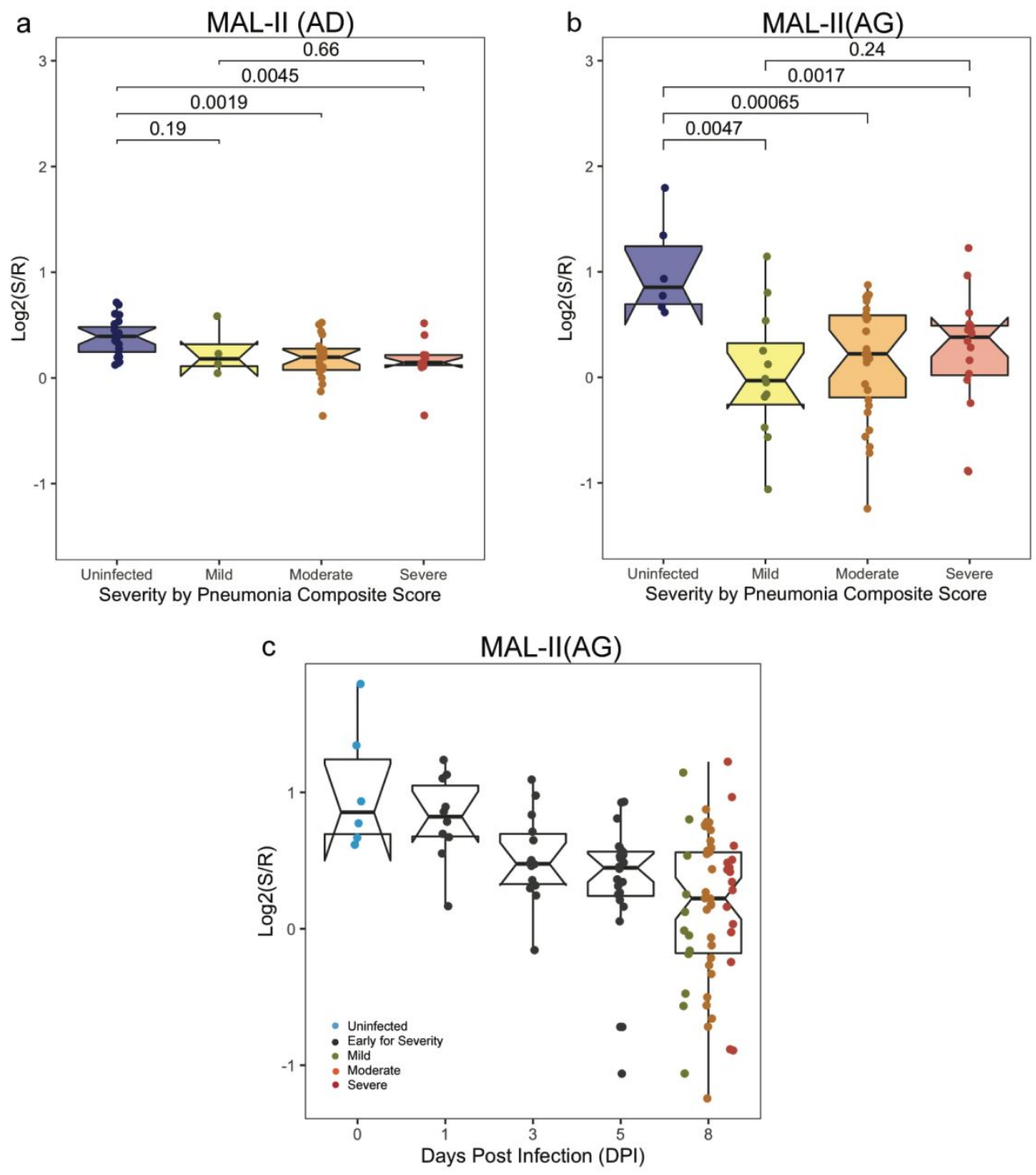

Figure S9. Impact of influenza infection on $\alpha-2,3$-sialosides in adult and aged ferrets. a) Severity study of $\alpha-2,3$-sialic acids probed by MAL-II at day 8 post-infection in adult ferrets (AD) is shown. Boxplots of median normalized $\log _{2}$ ratios (Sample $(\mathrm{S}) /$ Reference $(\mathrm{R})$ ) of ferret lung samples were ordered by severity defined by pneumonia composite score (PCS). Uninfected (blue): $\mathrm{n}=4,4$ samples per ferret. Day 8 post-infection: Mild (yellow), $\mathrm{n}=2,2$ samples per ferret; Moderate (orange), $\mathrm{n}=11,2$ samples per ferret; Severe (red), $\mathrm{n}=6,2$ samples per ferret. b) Severity study of $\alpha-2,3$-sialic acids probed by MAL-II at day 8 post-infection in aged ferrets (AG) is shown. Boxplots of median normalized $\log _{2}$ ratios (Sample $(\mathrm{S}) /$ Reference $(\mathrm{R})$ ) of ferret lung samples were ordered by severity defined by PCS. Uninfected (blue): $\mathrm{n}=3,2$ samples per ferret. Day 8 post-infection: Mild (yellow), $\mathrm{n}=6,2$ samples per ferret; Moderate (orange), $\mathrm{n}=15,2$ samples per ferret; Severe (red), $\mathrm{n}=8,2$ samples per ferret. P-value from Wilcoxon's t-test is shown for indicated pairs. c) Time-course analysis of $\alpha$-2,3-sialic acid levels probed by MAL-II following H1N1pdm09 infections ( $\mathrm{t}=0,1,3,5$ and 8 days) in aged animals (AG). Boxplot of median normalized $\log _{2}$ ratios (Sample $(\mathrm{S}) / \operatorname{Reference}(\mathrm{R})$ ) is shown. Disease severity is indicated by color (Uninfected: blue; Early for Severity: black; Mild: dark yellow; Moderate: orange, Severe: red). 

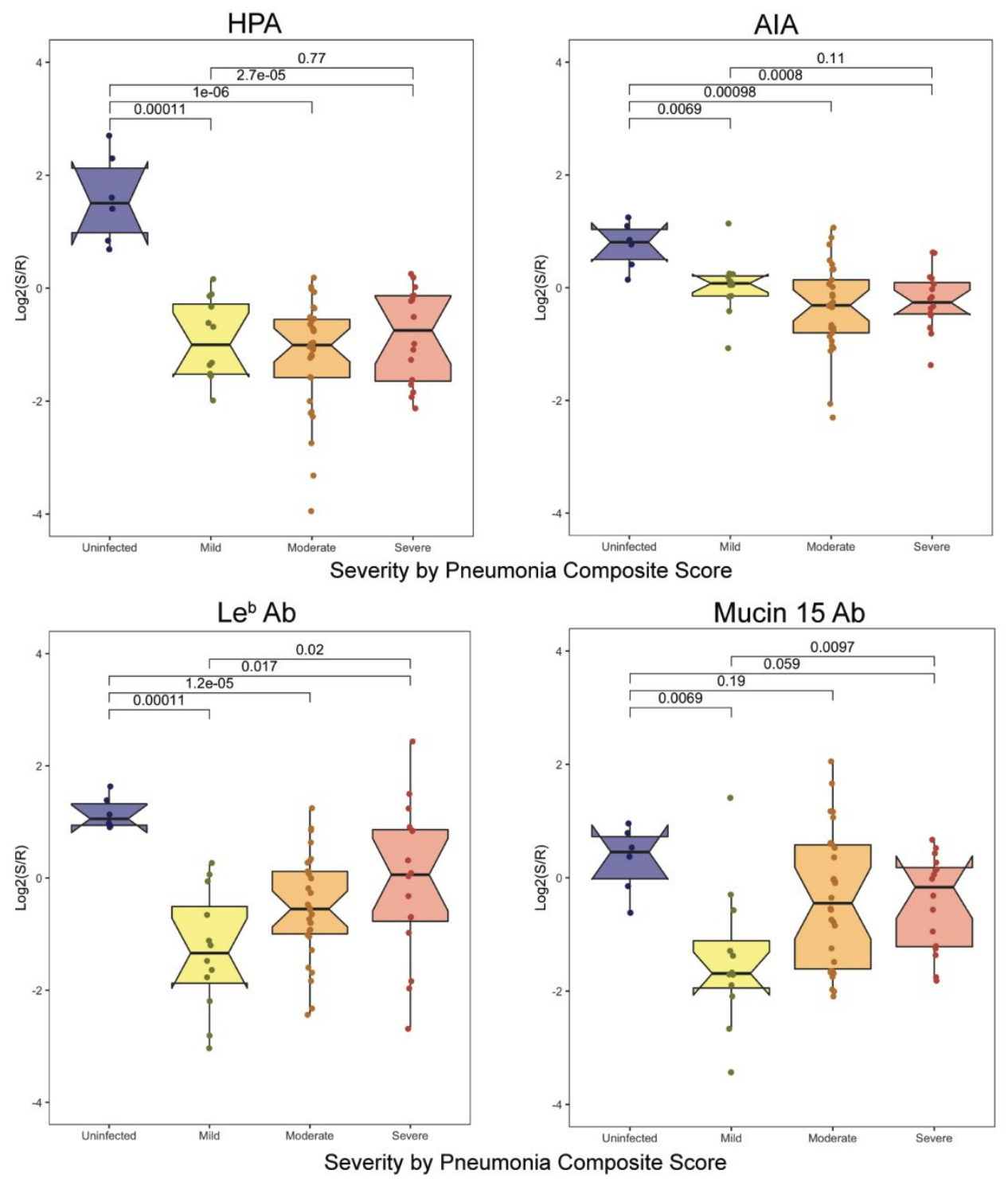

Figure S10. Impact of influenza infection on $\boldsymbol{O}$-linked glycans in aged ferrets. Severity study of $O$-linked glycans probed by HPA and AIA, Lewis structures by Le ${ }^{\mathrm{b}} \mathrm{Ab}$, and mucins by Mucin $15 \mathrm{Ab}$ at day 8 post-infection in aged ferrets is shown. Boxplots of median normalized $\log _{2}$ ratios (Sample (S)/Reference(R)) of ferret lung samples were ordered by severity defined by PCS. Uninfected (blue): $n=3,2$ samples per ferret. Day 8 infected: Mild (yellow), $n=6,2$ samples per ferret; Moderate (orange), $\mathrm{n}=15,2$ samples per ferret; Severe (red), $\mathrm{n}=8,2$ samples per ferret. $\mathrm{P}$-value from Wilcoxon's t-test is shown for indicated pairs. 

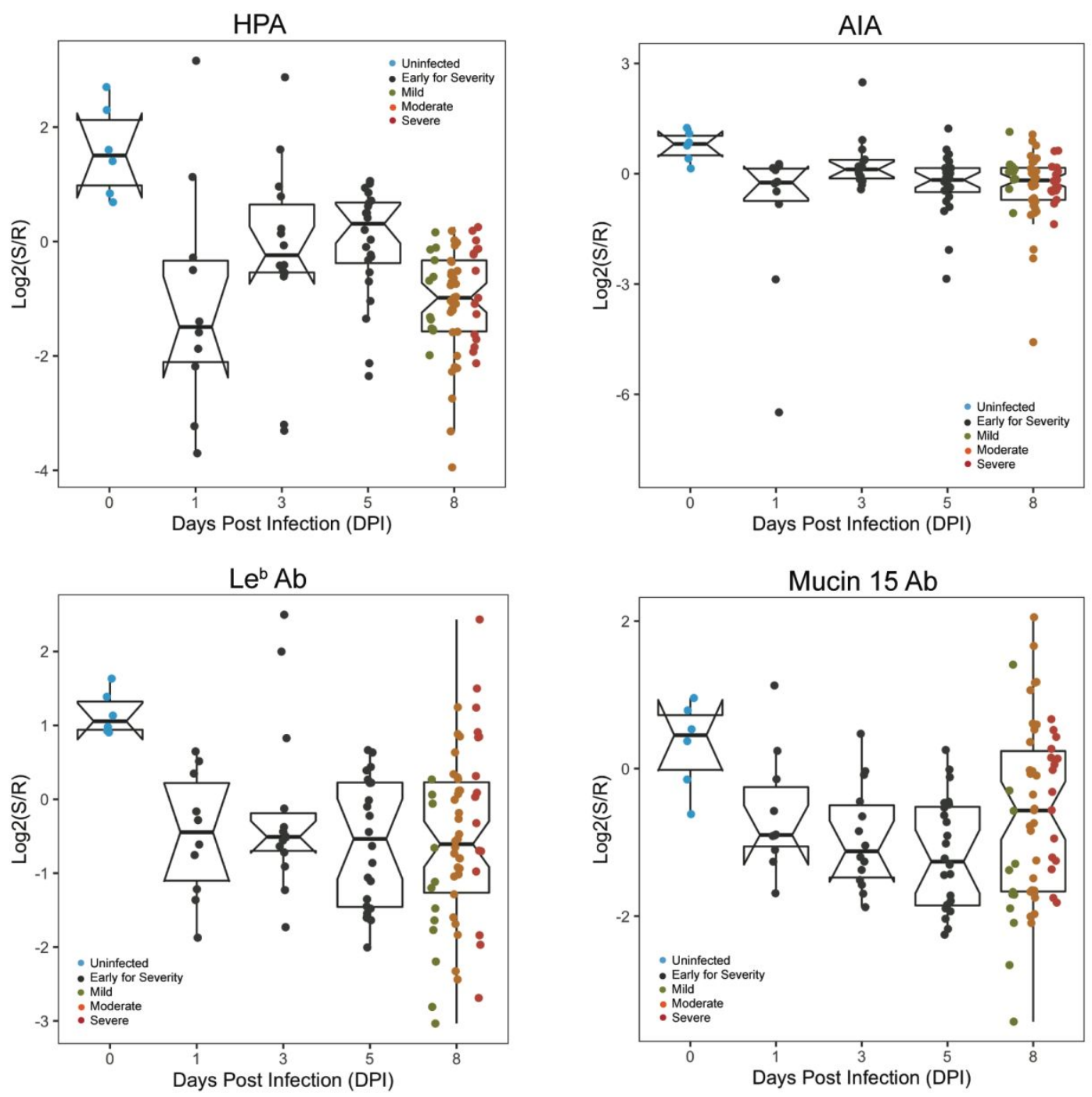

Figure S11. Dynamic changes in $\boldsymbol{O}$-linked glycans are observed upon infection in aged ferrets. Boxplot analysis of $O$-linked glycans probed by HPA and AIA, Lewis structures probed by Le ${ }^{b}$ $\mathrm{Ab}$, and mucins probed by Mucin $15 \mathrm{Ab}$ following H1N1pdm09 infections $(\mathrm{t}=0,1,3,5$ and 8 days). Boxplot of median normalized $\log _{2}$ ratios (Sample $(\mathrm{S}) / \operatorname{Reference}(\mathrm{R})$ ) is shown. Disease severity is indicated by color (Uninfected: blue; Early for Severity: black; Mild: dark yellow; Moderate: orange, Severe: red). 

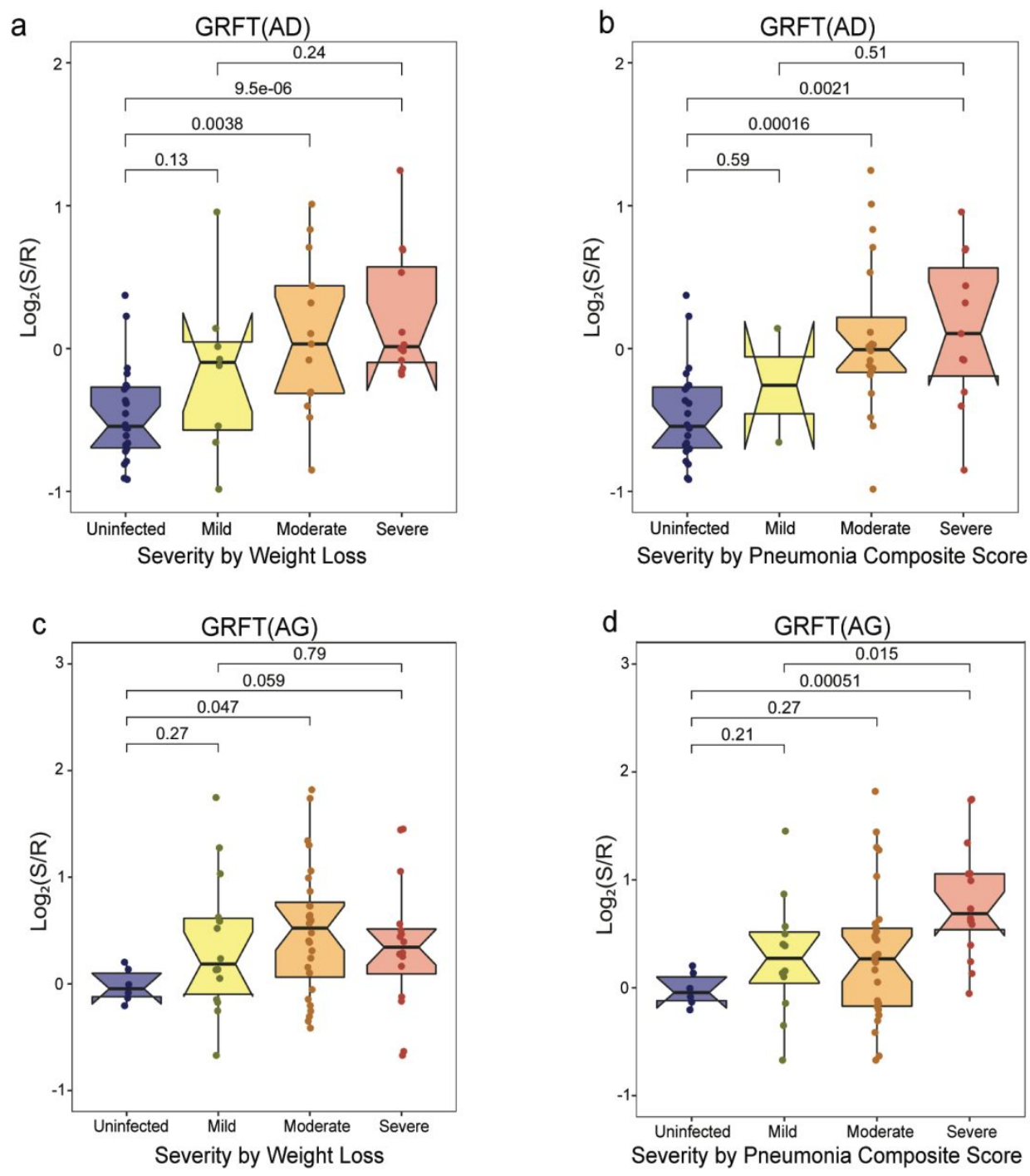

Figure S12. Comparison of severity metrics used in adult and aged ferrets. a) Boxplot analysis of lectin binding by GRFT (high-mannose) as a function of severity defined by weight loss in adult ferrets (AD). Uninfected (blue): $\mathrm{n}=4,4$ samples per ferret. Day 8 post-infection: Mild (yellow), $\mathrm{n}=5,2$ samples per ferret; Moderate (orange), $\mathrm{n}=7,2$ samples per ferret; Severe (red), $\mathrm{n}=7,2$ samples per ferret. b) Boxplot analysis of lectin binding by GRFT (high-mannose) as a function of severity defined by pneumonia composite score (PCS) in adult ferrets (AD). Uninfected (blue): $\mathrm{n}=4,4$ samples per ferret. Day 8 post-infection: Mild (yellow), $\mathrm{n}=2,2$ samples per ferret; Moderate (orange), $n=11,2$ samples per ferret; Severe (red), $n=6,2$ samples per ferret. c) Boxplot analysis of lectin binding by GRFT (high-mannose) as a function of severity defined by weight loss in aged ferrets (AG). Uninfected (blue): $\mathrm{n}=3,2$ samples per ferret. Day 8 post-infection: Mild (yellow), $\mathrm{n}=7$, samples per ferret; Moderate (orange), $\mathrm{n}=14,2$ samples per ferret; Severe (red), $\mathrm{n}=8,2$ samples per ferret. d) Boxplot analysis of lectin binding by GRFT (high-mannose) as a function of severity defined by PCS in aged ferrets (AG). Uninfected (blue): $n=3,2$ samples per ferret. Day 8 post-infection: Mild (yellow), $\mathrm{n}=6,2$ samples per ferret; Moderate (orange), $\mathrm{n}=15$, 2 samples per ferret; Severe (red), $\mathrm{n}=8,2$ samples per ferret. 

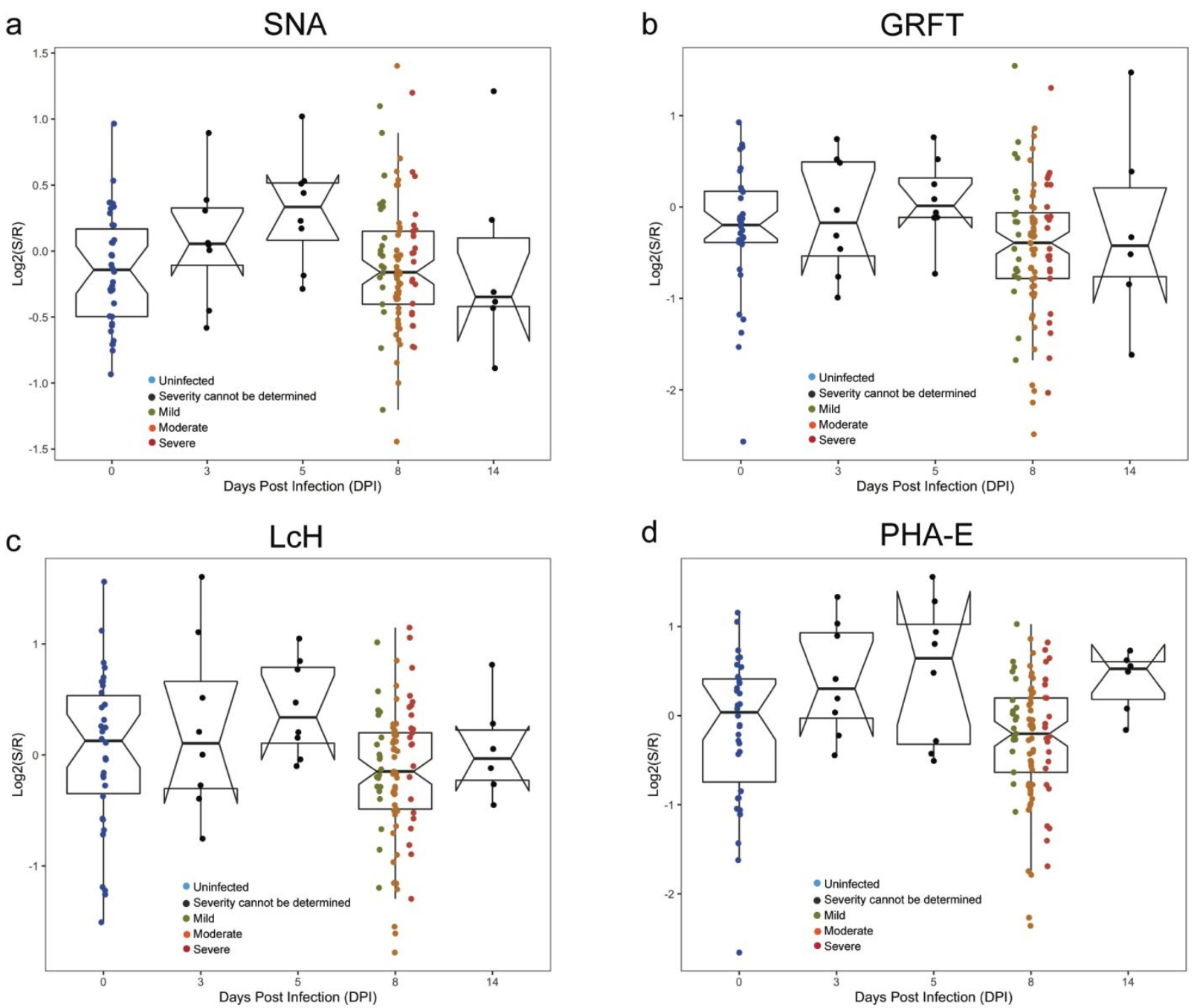

Figure S13. Glycan changes are observed early in the time-course study of newly weaned ferrets. a) Time-course analysis of $\alpha-2,6$-sialic acid probed by SNA following H1N1pdm09 infections ( $\mathrm{t}=0,3,5,8$ and 14 days). b) Time-course analysis of high/oligo-mannose probed by GRFT. c) Time-course analysis of core fucose probed by LcH. d) Time-course analysis of bisecting branching probed by PHA-E. Boxplot of median normalized $\log _{2}$ ratios (Sample (S)/Reference(R)) is shown. Disease severity is indicated by color (Uninfected: blue; Severity cannot be determined: black; Mild: dark yellow; Moderate: orange, Severe: red). 
Table S1. Lectins used in microarrays

\begin{tabular}{|c|c|c|c|c|}
\hline Lectin & Species/Origin & $\begin{array}{l}\text { Print Conc. } \\
(\mu \mathrm{g} / \mathrm{mL})\end{array}$ & $\begin{array}{l}\text { Rough } \\
\text { Specificity } \\
\text { /Inhibitory } \\
\text { monosaccharid } \\
\text { e }\end{array}$ & Vendor/Source \\
\hline $\mathrm{AAL}^{\mathrm{a}, \mathrm{b}, \mathrm{c}}$ & Aleuria aurantia & 1000 & Fucose & Vector \\
\hline $\mathrm{ACA}^{\mathrm{a}, \mathrm{b}, \mathrm{c}}$ & Amaranthus Caudatus & 1000 & $\begin{array}{l}\text { Gal- } \beta 1,3- \\
\text { GalNAc }\end{array}$ & Vector \\
\hline $\mathrm{AIA}^{\mathrm{a}, \mathrm{b}, \mathrm{c}}$ & Artocarpus integrifolia & 500 & $\beta 1,3-$ GalNAc & Vector/EY \\
\hline $\mathrm{AMA}^{\mathrm{a}, \mathrm{b}, \mathrm{c}}$ & Allium moly & 500 & Oligo mannose & EY \\
\hline $\begin{array}{l}\text { Anti-B.G.B [CLCP- } \\
\text { 19B] a }\end{array}$ & $\begin{array}{l}\text { MAb mouse IgM } \\
\text { [CLCP-19B] }\end{array}$ & undiluted & $\begin{array}{l}\text { Blood group B } \\
\text { antigen }\end{array}$ & Abcam \\
\hline Anti-B.G.H2 $2^{a, b, c}$ & $\begin{array}{l}\text { MAb mouse IgM [A46- } \\
\text { B/B10] }\end{array}$ & undiluted & $\begin{array}{l}\text { Blood group } \mathrm{H} 2 \\
\text { antigen }\end{array}$ & $\begin{array}{l}\text { Santa Cruz } \\
\text { Biotechnology }\end{array}$ \\
\hline Anti-CD 15[MY-1] ${ }^{a}$ & $\begin{array}{l}\text { Mab mouse IgM [MY- } \\
\text { 1] }\end{array}$ & undiluted & Lewis $\mathrm{X}$ & Abcam \\
\hline $\begin{array}{l}\text { Anti-B.G. Lewis A } \\
\text { [7LE] b,c }\end{array}$ & $\begin{array}{l}\text { MAb mouse IgG1 } \\
\text { [7LE] }\end{array}$ & undiluted & Lewis A & Abcam \\
\hline $\begin{array}{l}\text { Anti-B.G. Lewis B } \\
{[2-25 L E]^{\text {b,c }}}\end{array}$ & $\begin{array}{l}\text { MAb mouse IgM [2- } \\
\text { 25LE] }\end{array}$ & undiluted & Lewis B & Abcam \\
\hline Anti-B.G.Lewis $\mathrm{Y}^{\mathrm{b}, \mathrm{c}}$ & MAb mouse IgM & undiluted & Lewis Y & $\begin{array}{l}\text { Santa Cruz } \\
\text { Biotechnology }\end{array}$ \\
\hline 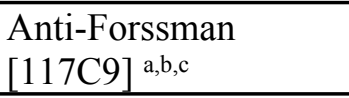 & MAb Rat IgM [117C9] & undiluted & $\begin{array}{l}\text { Forssman } \\
\text { Antigen }\end{array}$ & Abcam \\
\hline $\begin{array}{l}\text { Anti-Lewis B [T218] } \\
\mathrm{a}, \mathrm{b}, \mathrm{c}\end{array}$ & $\operatorname{IgM}[\mathrm{T} 218]$ & undiluted & Lewis B & Sigma \\
\hline $\begin{array}{l}\text { Anti-Lewis X [P12] } \\
\mathrm{a}, \mathrm{b}, \mathrm{c}\end{array}$ & MAb mouse IgM [P12] & undiluted & Lewis $\mathrm{X}$ & Abcam \\
\hline $\begin{array}{l}\text { Anti-Lewis Y [F3] } \\
\text { a,b,c }\end{array}$ & MAb mouse IgM [F3] & undiluted & Lewis $\mathrm{Y}$ & Abcam \\
\hline $\begin{array}{l}\text { Anti-MUC5AC } \\
\text { human [CLH2] }^{\mathrm{b}}\end{array}$ & $\begin{array}{l}\text { Mab mouse IgG1 } \\
\text { [CLH2] }\end{array}$ & undiluted & $\begin{array}{l}\text { human } \\
\text { MUC5AC }\end{array}$ & Sigma \\
\hline $\begin{array}{l}\text { Anti-MUC5AC } \\
\text { mouse }^{\text {b }}\end{array}$ & $\begin{array}{l}\text { Goat polyclonal to } \\
\text { mouse MUC5AC }\end{array}$ & undiluted & $\begin{array}{l}\text { mouse } \\
\text { MUC5AC }\end{array}$ & LSBio \\
\hline $\begin{array}{l}\text { Anti-Mucin } 15 \text { [H-5] } \\
\text { b,c }\end{array}$ & Mab mouse IgG1 [H-5] & undiluted & Mucin 15 & $\begin{array}{l}\text { Santa Cruz } \\
\text { Biotechnology } \\
\end{array}$ \\
\hline $\mathrm{AOL}^{\mathrm{a}, \mathrm{b}, \mathrm{c}}$ & Aspergillus oryzae & 1000 & Fucose & TCI America \\
\hline $\mathrm{APA}^{\mathrm{a}, \mathrm{b}, \mathrm{c}}$ & Abrus precatorius & 500 & $\begin{array}{l}\text { Gal- } \beta 1,3- \\
\text { GalNAc / Lac }\end{array}$ & EY \\
\hline $\mathrm{APP}$ a & $\begin{array}{l}\text { Aegopodium } \\
\text { podagraria }\end{array}$ & 1000 & $\begin{array}{l}\text { GalNAc / Lac / } \\
\text { Gal }\end{array}$ & EY \\
\hline $\mathrm{ASA}^{\mathrm{a}, \mathrm{b}, \mathrm{c}}$ & Allium sativum & 1000 & Mannose & EY \\
\hline $\mathrm{BDA}^{\mathrm{a}}$ & Bryonia dioica & 1000 & GalNAc / Lac & EY \\
\hline
\end{tabular}




\begin{tabular}{|c|c|c|c|c|}
\hline Blackbean ${ }^{a, b, c}$ & Blackbean crude & 1000 & GalNAc & EY \\
\hline $\mathrm{BPA}^{\mathrm{a}, \mathrm{b}, \mathrm{c}}$ & Bauhinia purpurea & 500 & $\begin{array}{l}\beta-\mathrm{Gal} / \beta- \\
\text { GalNAc }\end{array}$ & Vector \\
\hline $\mathrm{CA}^{\mathrm{a}, \mathrm{b}, \mathrm{c}}$ & Colchicum autumnale & 1200 & $\begin{array}{l}\text { Bi-antennary N- } \\
\text { linked glycans }\end{array}$ & EY \\
\hline $\mathrm{CAA}^{\mathrm{a}}$ & Caragana arborescens & 1000 & $\begin{array}{l}\text { Bi-antennary N- } \\
\text { linked glycans }\end{array}$ & EY \\
\hline Calsepa ${ }^{b, c}$ & Calystegia sepium & 1000 & $\begin{array}{l}\text { Bisecting N- } \\
\text { linked glycans }\end{array}$ & EY \\
\hline $\mathrm{CCA}^{\mathrm{a}}$ & Cancer antennarius & 1000 & $\begin{array}{l}\text { 9-O-Acetly } \\
\text { sialylation / 4-O- } \\
\text { Acetyl } \\
\text { sialylation }\end{array}$ & EY \\
\hline Cholera Toxin ${ }^{b}$ & Vibrio cholerae & 1000 & $\begin{array}{l}\text { GM1 } \\
\text { ganglioside }\end{array}$ & Sigma \\
\hline $\operatorname{Con} \mathrm{A}^{\mathrm{b}, \mathrm{c}}$ & Canavalia ensiformis & 1000 & $\begin{array}{l}\text { Tri-mannose } \\
\text { core }\end{array}$ & EY/Vector \\
\hline $\mathrm{CSA}^{\mathrm{a}, \mathrm{b}, \mathrm{c}}$ & Cystisus scoparius & 1000 & $\begin{array}{l}\text { Terminal } \\
\text { GalNAc }\end{array}$ & EY \\
\hline $\operatorname{DBA}^{\mathrm{a}, \mathrm{b}, \mathrm{c}}$ & Dolichos Biflorus & 1000 & GalNAc & Vector \\
\hline $\operatorname{diCBM} 40$ a,b,c & $\begin{array}{l}\text { engineered NanI from } \\
\text { Clostridium } \\
\text { perfringens } \\
\end{array}$ & 1000 & $\alpha$ Sialylation & $\begin{array}{l}\text { Generated in } \\
\text { house }\end{array}$ \\
\hline $\mathrm{DSA}^{\mathrm{a}, \mathrm{b}, \mathrm{c}}$ & Datura stramonium & 500 & LacNAc & EY/Vector \\
\hline $\mathrm{ECA}^{\mathrm{a}, \mathrm{b}, \mathrm{c}}$ & Erythrina cristagalli & 1000 & LacNAc & Vector \\
\hline EEL/EEA ${ }^{a, b, c}$ & Eunonymus europaeus & 1000 & Blood Group B & Vector/EY \\
\hline GafD a,b,c & $\begin{array}{l}\text { recombinant GafD } \\
\text { from Escherichia coli } \\
\end{array}$ & 1000 & GlcNAc & $\begin{array}{l}\text { Generated in } \\
\text { house }\end{array}$ \\
\hline GNA/GNL a,b c & Galanthus nivalis & 1500 & Oligo mannose & Vector/EY \\
\hline $\mathrm{GHA}^{\text {a }}$ & Glechoma hederacea & 500 & GalNac & EY \\
\hline GS-I a,b,c & Griffonia simplicifoia-I & 1000 & $\alpha-\mathrm{Gal} / \mathrm{Lac}$ & Vector/EY \\
\hline GS-II a,b,c & $\begin{array}{l}\text { Griffonia simplicifoia- } \\
\text { II }\end{array}$ & 1000 & GlcNAc & Vector \\
\hline GS-IB4 a,b,c & $\begin{array}{l}\text { Griffonia simplicifoia-I, } \\
\text { isolectin B4 }\end{array}$ & 2000 & Gal & Vector \\
\hline H84T b,c & Banana lectin & 1000 & High mannose & $\begin{array}{l}\text { Gift from Dr. } \\
\text { David } \\
\text { Markovitz }\end{array}$ \\
\hline $\mathrm{HAA}^{\mathrm{a}, \mathrm{b}, \mathrm{c}}$ & Homarus americanus & 1000 & $\begin{array}{l}\text { Terminal } \\
\text { GalNAc }\end{array}$ & EY \\
\hline
\end{tabular}




\begin{tabular}{|c|c|c|c|c|}
\hline $\mathrm{HHL}^{\mathrm{a}, \mathrm{b}, \mathrm{c}}$ & Hippeastrum Hybrid & 1500 & $\begin{array}{l}\text { Oligo/High } \\
\text { mannose }\end{array}$ & Vector \\
\hline $\mathrm{HPA}^{\mathrm{a}, \mathrm{b}, \mathrm{c}}$ & Helix pomatia & 1000 & Blood Group A & Sigma/EY \\
\hline $\operatorname{IRA}^{\mathrm{a}}$ & Iris Hybrid & 1000 & GalNAc / Lac & EY \\
\hline $\mathrm{LBA}^{\mathrm{a}}$ & Phaseolus lunatus & 1000 & Blood Group A & EY \\
\hline LAA $^{b}$ & Laburnum alpinum & 900 & GlcNAc & EY \\
\hline $\mathrm{LcH}^{\mathrm{a}, \mathrm{b}, \mathrm{c}}$ & Lens Culinaris & 1000 & Core Fucose & Vector \\
\hline LEA/LEL ${ }^{\mathrm{a}, \mathrm{b}, \mathrm{c}}$ & $\begin{array}{l}\text { Lycopersicon } \\
\text { esculentum }\end{array}$ & 1000 & GlcNAc & Vector/EY \\
\hline $\mathrm{LFA}^{\mathrm{a}}$ & Limax flavus & 500 & $\alpha$ Sialylation & EY \\
\hline Lotus a,b,c & Lotus tetragonolobus & 1000 & Fucose & Vector \\
\hline $\mathrm{MAA}^{\mathrm{a}}$ & Maackia amurensis & 500 & $\begin{array}{l}\text { Sialylation/Sulfa } \\
\text { tion }\end{array}$ & EY \\
\hline MAL-I ${ }^{a, b, c}$ & Maackia amurensis-I & 2000 & $\begin{array}{l}\text { Sialylation/Sulfa } \\
\text { tion }\end{array}$ & Vector \\
\hline MAL-II a,b,c & Maackia amurensis-II & 2000 & $\begin{array}{l}\text { Sialylation/Sulfa } \\
\text { tion }\end{array}$ & Vector \\
\hline MNA-G a,b,c & $\begin{array}{l}\text { Morus nigra Morniga } \\
G\end{array}$ & 1000 & GalNAc & EY \\
\hline MNA-M a,b,c & $\begin{array}{l}\text { Morus nigra Morniga } \\
M\end{array}$ & 1000 & $\begin{array}{l}\text { Oligo mannose / } \\
\text { Gal }\end{array}$ & EY \\
\hline MPA/MPL a,b,c & Maclura pomifera & 1000 & $\beta 1,3-$ GalNAc & Vector \\
\hline newBR6 ${ }^{b}$ & $\begin{array}{l}\text { unknown (from } \\
\text { unpublished work) }\end{array}$ & 480 & $\begin{array}{l}\text { under } \\
\text { investigation }\end{array}$ & $\begin{array}{l}\text { Gift from Dr. } \\
\text { Barbara Bensing }\end{array}$ \\
\hline $\mathrm{NPA}^{\mathrm{a}, \mathrm{b}, \mathrm{c}}$ & $\begin{array}{l}\text { Narcissus } \\
\text { pseudonarcissus }\end{array}$ & 1000 & Oligo mannose & Vector \\
\hline PA-I ${ }^{a, b, c}$ & $\begin{array}{l}\text { Pseudomonas } \\
\text { aeruginosa }\end{array}$ & 1000 & Gal & Sigma \\
\hline PA-IL a & bacteria & 1000 & GalNAc & $\begin{array}{l}\text { Generated in } \\
\text { house }\end{array}$ \\
\hline PHA-E ${ }^{a, b, c}$ & $\begin{array}{l}\text { Phaseolus vulgaris } \\
\text { Erythroagglutinin }\end{array}$ & 1000 & $\begin{array}{l}\text { Bisecting } \\
\text { GlcNAc }\end{array}$ & $\begin{array}{l}\text { Vector/EY/Sigm } \\
\mathrm{a}\end{array}$ \\
\hline PHA-L ${ }^{a, b, c}$ & $\begin{array}{l}\text { Phaseolus vulgaris } \\
\text { Leukoagglutinin }\end{array}$ & 1000 & $\begin{array}{l}\beta 1,6 \text { Branching } \\
\text { N-Link glycans }\end{array}$ & Vector/EY \\
\hline $\mathrm{PMA}^{\mathrm{a}}$ & $\begin{array}{l}\text { Polygonatum } \\
\text { multiflorum }\end{array}$ & 500 & Oligo mannose & EY \\
\hline $\mathrm{PNA}^{\mathrm{a}, \mathrm{b}, \mathrm{c}}$ & Arachis hyogaea & 1000 & $\begin{array}{l}\text { Gal- } \beta 1,3- \\
\text { GalNAc }\end{array}$ & Vector/EY \\
\hline $\operatorname{PSA}^{\mathrm{a}, \mathrm{b}, \mathrm{c}}$ & Pisum sativum & 1000 & Core Fucose & Vector \\
\hline PSL a & Polyporus squamosus & 1000 & $\alpha 2,6$ sialylation & EY \\
\hline PTA $^{a, b, c}$ & $\begin{array}{l}\text { Psophocarpus } \\
\text { tetragonolobus }\end{array}$ & 500 & Blood Groups & EY \\
\hline
\end{tabular}




\begin{tabular}{|c|c|c|c|c|}
\hline PTL-I a,b,c & $\begin{array}{l}\text { Psophocarpus } \\
\text { tetragonolobus-I }\end{array}$ & 1500 & Blood Group A & Vector \\
\hline PTL-II a,b,c & \begin{tabular}{|l} 
Psophocarpus \\
tetragonolobus-II
\end{tabular} & 1000 & $\alpha 2$ Fucose & Vector \\
\hline RCA120 a,b,c & $\begin{array}{l}\text { Ricinus Communis } \\
\text { Agglutinin I }\end{array}$ & 1000 & $\mathrm{Gal} / \mathrm{Lac}$ & Vector \\
\hline $\mathrm{rCVN}^{\mathrm{a}}$ & \begin{tabular}{|l|} 
recombinant \\
Cyanovirin \\
\end{tabular} & 1000 & High mannose & $\begin{array}{l}\text { Gift from Dr. } \\
\text { Barry O'Keefe }\end{array}$ \\
\hline rGRFT $^{\mathrm{a}, \mathrm{b}, \mathrm{c}}$ & recombinant Griffithsin & 1000 & High mannose & $\begin{array}{l}\text { Gift from Dr. } \\
\text { Barry O'Keefe }\end{array}$ \\
\hline Ricin B Chain a,b,c & Ricinus communis & 1000 & Gal & Vector \\
\hline $\mathrm{RPA}^{\mathrm{a}, \mathrm{b}, \mathrm{c}}$ & Robinia pseudoacacia & 500 & $\begin{array}{l}\text { Complex N-link } \\
\text { glycans }\end{array}$ & EY \\
\hline $\mathrm{rSVN}$ a,b & recombinant Scytovirin & 1000 & High mannose & $\begin{array}{l}\text { Gift from Dr. } \\
\text { Barry O'Keefe }\end{array}$ \\
\hline $\mathrm{SBA}^{\mathrm{a}, \mathrm{b}, \mathrm{c}}$ & Glycine $\max$ & 1000 & LacdiNAc & Vector \\
\hline SJA ${ }^{a, b, c}$ & Sophora japonica & 1000 & LacdiNAc & Vector \\
\hline SK $1^{b}$ & \begin{tabular}{|l|} 
Streptococcus \\
sanguinis $S K 1$ \\
\end{tabular} & 1800 & $\alpha 2,3$ sialylation & $\begin{array}{l}\text { Gift from Dr. } \\
\text { Barbara Bensing }\end{array}$ \\
\hline SK678 b & \begin{tabular}{|l|} 
Streptococcus \\
sanguinis SK678 \\
\end{tabular} & 450 & $\alpha 2,3$ sialylation & $\begin{array}{l}\text { Gift from Dr. } \\
\text { Barbara Bensing }\end{array}$ \\
\hline SLBR-B b,c & $\begin{array}{l}\text { Streptococcus gordonii } \\
\text { M99 }\end{array}$ & 1000 & $\alpha 2,3$ sialylation & $\begin{array}{l}\text { Gift from Dr. } \\
\text { Barbara Bensing }\end{array}$ \\
\hline SLBR-H ${ }^{b, c}$ & $\begin{array}{l}\text { Streptococcus gordonii } \\
\text { DL1 }\end{array}$ & 2000 & $\alpha 2,3$ sialylation & $\begin{array}{l}\text { Gift from Dr. } \\
\text { Barbara Bensing }\end{array}$ \\
\hline SLBR-N b,c & \begin{tabular}{|l|} 
Streptococcus gordonii \\
UB10712 \\
\end{tabular} & 1000 & $\alpha 2,3$ sialylation & $\begin{array}{l}\text { Gift from Dr. } \\
\text { Barbara Bensing }\end{array}$ \\
\hline $\mathrm{SNA}^{\mathrm{a}, \mathrm{b}, \mathrm{c}}$ & Sambucus nigra & $500 / 1000$ & $\alpha 2,6$ sialylation & Vector/Sigma \\
\hline SNA-II ${ }^{a, b, c}$ & Sambucus nigra-II & 1000 & $\begin{array}{l}\alpha 2 \text { Fucose /oligo } \\
\text { mannose }\end{array}$ & EY \\
\hline STA/STL ${ }^{a, b, c}$ & Solanus tuberosum & 500 & GlcNAc & Vector \\
\hline TJA-I a,b,c & $\begin{array}{l}\text { Trichosanthes } \\
\text { japonica-I }\end{array}$ & 1000 & $\alpha 2,6$ sialylation & TCI \\
\hline TJA-II a,b,c & $\begin{array}{l}\text { Trichosanthes } \\
\text { japonica-II }\end{array}$ & 1000 & $\alpha 2$ Fucose & $\begin{array}{l}\text { NorthStar } \\
\text { Bioproducts/Ani } \\
\text { ara Diagnostica }\end{array}$ \\
\hline $\mathrm{TL}^{\mathrm{a}, \mathrm{b}, \mathrm{c}}$ & Tulipa sp. & 700 & GlcNAc & EY \\
\hline $\mathrm{UDA}^{\mathrm{a}, \mathrm{b}}$ & Urtica dioica & 1000 & $\begin{array}{l}\text { GlcNAc / Oligo } \\
\text { mannose }\end{array}$ & EY \\
\hline UEA-I a,b,c & Ulex europaaeus-I & 1000 & $\alpha 2$ Fucose & Vector \\
\hline UEA-II a,b,c & Ulex europaaeus-II & 2000 & GlcNAc & Vector \\
\hline VFA $^{\mathrm{a}, \mathrm{b}, \mathrm{c}}$ & Vicia faba & 1000 & GlcNAc & EY \\
\hline
\end{tabular}




\begin{tabular}{|l|l|l|l|l|} 
VVA a,b,c & Vicia villosa & 1000 & $\begin{array}{l}\text { Terminal } \\
\text { GalNAc }\end{array}$ & Vector/EY \\
\hline VVA(man) $)^{\text {a,b,c }}$ & Vicia villosa & 500 & Mannose & Vector/EY \\
\hline WFA $^{\text {a,b,c }}$ & Wisteria floribunda & 1000 & GalNAc- $\beta 1,4$ & Vector \\
\hline WGA $^{\text {a,b,c }}$ & Triticum vulgare & 1000 & GlcNAc & Vector/EY \\
\hline X408 ${ }^{\mathrm{b}}$ & $\begin{array}{l}\text { unknown (from } \\
\text { unpublished work) }\end{array}$ & 1200 & $\begin{array}{l}\text { under } \\
\text { investigation }\end{array}$ & $\begin{array}{l}\text { Gift from Dr. } \\
\text { Barbara Bensing }\end{array}$ \\
\hline
\end{tabular}

a : lectins printed in uninfected experiment

${ }^{b}$ : lectins printed in newly weaned experiment

$\mathrm{c}$ : lectins printed in aged experiment 
Table S2. Lectin Microarray Information

\section{Description}

1. Sample: Glycan-containing sample (e.g. glycan, glycoprotein, cell lysate etc.)

\begin{tabular}{|l|l|}
\hline Description of Sample & Glycoproteins extracted from whole lung tissues \\
\hline $\begin{array}{l}\text { Sample preparation } \\
\text { protocol }\end{array}$ & $\begin{array}{l}\text { Ferret lung tissues are washed with PBS supplemented with } \\
\text { protease inhibitor cocktails. The solutions are sonnicated on ice } \\
\text { until completely homogenous and then centrifuged to collect } \\
\text { supernatants prior to labeling. }\end{array}$ \\
\hline $\begin{array}{l}\text { Labeling protocol for } \\
\text { sample detection }\end{array}$ & $\begin{array}{l}\text { Samples are labelled with Alexa Fluor 555-NHS (Thermo } \\
\text { Fisher). }\end{array}$ \\
\hline $\begin{array}{l}\text { Two-color reference (if } \\
\text { used) }\end{array}$ & $\begin{array}{l}\text { A pooled reference samples are labelled with Alexa Fluor 647- } \\
\text { NHS (Thermo Fisher). }\end{array}$ \\
\hline Assay protocol & $\begin{array}{l}\text { Lectin microarrays are blocked with blocking buffer for one } \\
\text { hour at room temperature. Slides are rinsed twice with PBST } \\
\text { (0.005\%) and once with PBS, then dry the slide using a slide } \\
\text { spinner. Each slide was mounted on a 24-well format } \\
\text { hybridization cassette (Arrayit), in which each well contains a } \\
\text { subarray. To each well, add equal amounts of samples and } \\
\text { universal reference, and dilute with PBS and PBST (0.2\%) to } \\
\text { reach the final volume (150uL). Incubate the slides on an orbital } \\
\text { shaker for two hours at room temperature in the dark. After } \\
\text { hybridization, wash the arrays with PBST (0.005\%) twice for } \\
\text { ten minutes, and twice for five minutes. Once finished, remove } \\
\text { the slides from the cassette, and immerse the slides in ultrapure } \\
\text { water, and dry the slides using a slide spinner. }\end{array}$ \\
\hline
\end{tabular}

\section{Lectin Library}

General description of the lectin library used in the array

List of lectins and glycan binding proteins, source, concentration and buffer

Modification of lectins (e.g. biotin) if any. Lectin microarrays are generated in house.

Please see Supplemental Table 1.

\section{Immobilization Surface; e.g., Microarray Slide}




\begin{tabular}{|l|l|}
\hline Immobilization surface & Nexterion Slide H Barcoded 3D Hydrogel Coated \\
\hline Manufacturer & Schott North America \\
\hline $\begin{array}{l}\text { Custom preparation of } \\
\text { surface }\end{array}$ & N/A \\
\hline 4. Array Production & $\begin{array}{l}\text { Nano-Plotter 2.1 piezoelectric printer (GeSim, Germany) with } \\
\text { cooled microwell plate holder and cooled printing deck }\end{array}$ \\
\hline Description of Arrayer & Three replicates of each lectin are printed onto each subarray. \\
\hline Lectin deposition & $\begin{array}{l}\text { Dilute lectins to the pre-determined concentrations in the print } \\
\text { buffer (final concentration of print buffer: 0.01\% Tween-20, } \\
\text { 1mM monosaccharide in PBS; Please see Supplemental Table } \\
\mathbf{1} \text { for the concentrations of lectins). Load the mixed solution to } \\
\text { the microplate. Before printing, check the humidity of the print } \\
\text { chamber. The humidity should be kept around 50\% during the } \\
\text { entire printing. Ensure both microwell plate holder and printing } \\
\text { deck are cooled. Adjust the cooling temperature based on } \\
\text { ambient temperature and the temperature of the cooled slide } \\
\text { deck surface, preventing moisture building up inside the print } \\
\text { chamber. Once printing is complete, allow the slides to dry for } \\
\text { at least one hour. }\end{array}$ \\
\hline Printing conditions & $\begin{array}{l}\text { For each microarray, it contains 24 subarrays (3 columns and 8 } \\
\text { rows). In each subarray, triplicates of a lectin are printed, and } \\
\text { for a row with five lectins, the spot layout should be 15 columns. } \\
\text { The row number depends on how many lectin probes are printed } \\
\text { on the arrays (i.e., 110 lectins require 22 rows). }\end{array}$ \\
\hline Instrument settings & $\begin{array}{l}\text { Well-characterized glycoproteins including fetuin, asialofetuin } \\
\text { and RNase B are used for quality assurances of the printed } \\
\text { microarrays. }\end{array}$ \\
\hline $\begin{array}{l}\text { 5. Detector and Data Processing } \\
\text { cytometer) }\end{array}$ & $\begin{array}{l}\text { Preview the slide to adjust photomultiplier gain (PMT) for each } \\
\text { channel (Alexa Fluor-555: 532nm, Alexa Fluor-647: 635nm) so } \\
\text { that the signals are not saturated and within the linear detection } \\
\text { range. }\end{array}$ \\
\hline & $\begin{array}{l}\text { Fluorescent Slide Scanner Genepix 4300A (Molecular Devices) } \\
\text { Array }\end{array}$ \\
\hline
\end{tabular}




\begin{tabular}{|l|l|}
\hline Image analysis software & GenePix Pro 7 (Molecular Devices) \\
\hline $\begin{array}{l}\text { Data processing and } \\
\text { statistical analysis }\end{array}$ & $\begin{array}{l}\text { Extracted data is processed for quality checks using Grubbs } \\
\text { outlier test with } \alpha=0.05 . \mathrm{Log}_{2} \text { values of the average signals are } \\
\text { median-normalized over the individual subarray in each } \\
\text { channel. }\end{array}$ \\
\hline 6. Lectin Microarray Data Presentation \\
\hline $\begin{array}{l}\text { Data presentation and } \\
\text { interpretation }\end{array}$ & $\begin{array}{l}\text { Hierarchical clustering of the processed data is performed using } \\
\text { Pearson Correlation coefficient, and visualized with Multi- } \\
\text { experiment Viewer (MeV, v4.8, TM4 Microarray Software } \\
\text { Suite). If a lectin's SNR (signal-to-noise ratio) }<3 \text { for more than } \\
\text { one third of the total samples, then this lectin is considered as } \\
\text { inactive and excluded from the list. P-values are calculated } \\
\text { using nonparametric statistical tests, which are generated by R } \\
\text { (v3.6.1). }\end{array}$ \\
\hline 7. Data Location & 10.7303/syn22176606 \\
\hline Data Location &
\end{tabular}

